[0212-7199 (2005) 22: 6; pp 271-274] ANALES DE MEDICINA INTERNA Copyright $@ 2005$ ARAN EDICIONES, S.L.

An. MED. INTERNA (Madrid) Vol. 22, N. ${ }^{\circ} 6$, pp. 271-274, 2005

\title{
La anemia es un factor pronóstico de mortalidad en la insuficiencia cardiaca
}

\author{
G. GARCÍA DE CASASOLA, C. CÁRDENAS FRANCO, A. VEGAS SERRANO, \\ M. A. HORNERO IZQUIERDO, C. GUIJARRO HERRAIZ, A. ZAPATERO GAVIRIA
}

Unidad de Medicina Interna. Fundación Hospital Alcorcón. Alcorcón. Madrid

ANAEMIA PREDICTS MORTALITY IN HEART FAILURE

\section{RESUMEN}

Antecedentes: La anemia es frecuente en los enfermos con insuficiencia cardíaca (IC). Son pocos los estudios en los que se valora el impacto de la misma sobre la mortalidad en estos pacientes.

Objetivo: Valorar la prevalencia de la anemia entre los enfermos con IC y su posible relación con la mortalidad.

Material y métodos: Estudiamos las historias clínicas informatizadas de 272 enfermos que ingresaron en la Unidad de Medicina Interna de la Fundación Hospital Alcorcón con el diagnóstico principal de IC según los criterios de Framingham entre julio y diciembre de 2003. Realizamos análisis de regresión logística uni y multifactorial para valorar los factores pronósticos de mortalidad.

Resultados: La mayoría de los enfermos eran mujeres (71\%), la edad media fue 82,1 \pm 9 años, $106(39,9 \%)$ tenían anemia (niveles de hemoglobina séricos $<12 \mathrm{~g} / \mathrm{dL}$ ), 69 (25\%) tenían insuficiencia renal (niveles de creatitina séricos $>1,5 \mathrm{mg} / \mathrm{dL}$ ) y 154 pacientes $(57 \%)$ tenían fibrilación auricular. Hubo 41 defunciones (15\%). La anemia fue más frecuente y los niveles de hemoglobina eran más bajos en los enfermos que fallecieron $(65 \%$ frente $36 \%$ y $11,2 \pm 2,4 \mathrm{~g} / \mathrm{dL}$ frente $12,6 \pm 2,1 \mathrm{~g} / \mathrm{dL}$, respectivamente, $\mathrm{p}<0,001$ para ambos). El aumento de los niveles de creatinina séricos también se asociaron con la mortalidad $(1,8 \pm 0,8$ vs $1,3 \pm 0,8$ $\mathrm{mg} / \mathrm{dL} \mathrm{p}<0,001)$. En el análisis unifactorial, la edad, el sexo, la fibrilación auricular y la etiología de la IC no se asociaron con la mortalidad. En el análisis de regresión logística multifactorial los factores pronósticos de mortalidad fueron la hemoglobina (odds ratio [OR] 0,78 por $\mathrm{g} / \mathrm{dL}$ intervalo de confianza [IC] 95\% 0,66-0,923 $\mathrm{p}<0,01$ ), la clase functional de la New York Heart Association (OR 2,2, IC 95\% 1,2-3,9 p <0,01) y la creatinina sérica (OR 1,5 por mg/dL, IC $95 \% 0,98-2,31 \mathrm{p}=0,06$ ).

Conclusiones: La anemia es un problema frecuente entre los enfermos con IC y constituye un factor pronóstico independiente de mortalidad.

PALABRAS CLAVE: Anemia. Insuficiencia cardiaca. Insuficiencia renal. Mortalidad.

\section{ABSTRACT}

Background: Anaemia frequently coexists with heart failure. Few studies have examined the impact of anaemia on mortality in this population.

Objective: To assess the prevalence of anaemia in patients with heart failure in an Internal Medicine Unit and its potential effects on mortality.

Material and methods: We evaluated the electronic medical records of 272 patients with heart failure that fulfilled the criteria admitted to the Internal Medicine Unit of Fundación Hospital Alcorcón (Madrid, Spain) between July and December 2003. Uni and multivariate logistic regression analysis for predictors of mortality.

Results: Most patients were women (71\%), mean age was $82,1 \pm 9$ years, $106(39,9 \%)$ had anaemia (serum haemoglobin levels $<12 \mathrm{~g} / \mathrm{dL}$ ), $69(25 \%)$ had renal failure (serum creatinine levels $>1.5 \mathrm{mg} / \mathrm{dL}$ and 154 patients $(57 \%)$ had atrial fibrillation. There were 41 deaths $(15 \%)$. The frequency of anaemia was higher and the haemoglobin levels were lower in the patients who died $(65 \%$ vs $36 \%$, and $11.2 \pm 2.4 \mathrm{~g} / \mathrm{dL}$ vs $12.6 \pm 2.1$ $\mathrm{g} / \mathrm{dL}, p<0.001$ for both). Increased serum creatinine was also associated with mortality $(1.8 \pm 0.8$ vs $1.3 \pm 0.8 \mathrm{mg} / \mathrm{dL} p<0.001)$. Age, gender, atrial fibrillation or the aetiology of heart failure were not associated with mortality in univariate analysis. In contrast, by multivariate logistic regression analysis, haemoglobin (odds ratio [OR] 0,78 per g/dL, 95\% confidence interval [CI] 0.66- 0.923 $p<0.01)$, New York Heart Association functional classification (OR 2.2, 95\% CI 1.2-3.9 $p<0.01$ ), and serum creatinine (OR $1.5 \mathrm{per} \mathrm{mg} / \mathrm{dL}$, 95\% CI 0.98-2.31 $p=0.06)$ were independent predictors of mortality.

Conclusions: Anaemia is a frequent problem among patients with heart failure and it is a significant independent risk factor for death.

KEY WORDS: Anaemia. Heart failure. Congestive. Kidney failure. Hospital mortality.

García de Casasola G, Cárdenas Franco C, Vegas Serrano A, Hornero Izquierdo MA, Guijarro Herraiz C, Zapatero Gaviria A. La anemia es un factor pronóstico de mortalidad en la insuficiencia cardiaca. An Med Interna (Madrid) 2005; 22: 271-274. 


\section{INTRODUCCIÓN}

La insuficiencia cardiaca (IC) es una de las principales causas de morbilidad y mortalidad en los países industrializados y constituye uno de los motivos más frecuentes de hospitalización entre las personas de más de 65 años. Se calcula que en la Europa comunitaria unos 7 millones de personas tienen IC (1).

La anemia es una complicación relativamente frecuente de muchas enfermedades crónicas. Sin embargo, su relación con la IC sólo se ha tenido en consideración en los últimos años (2-5). La prevalencia de la anemia es variable dependiendo del tipo de población con IC seleccionada. En muchas de las series más del $25 \%$ de los enfermos con IC tienen anemia $(3,6,7)$, porcentaje superior al observado en la población general de edad similar (8). Asimismo, son pocos los estudios en los que se valora la importancia pronóstica de la anemia entre los pacientes con IC $(2,9)$. El objetivo de nuestro estudio ha sido valorar la prevalencia de anemia entre los enfermos que ingresan por IC en una unidad de Medicina Interna y analizar su efecto potencial sobre la mortalidad durante el ingreso.

\section{MATERIAL Y MÉTODO}

De forma retrospectiva revisamos las historias clínicas informatizadas de todos los enfermos ingresados con el diagnóstico principal de IC ("Clasificación Internacional de Enfermedades" [ICD]-9, código 428.x) en la unidad de Medicina Interna de la Fundación Hospital Alcorcón entre julio y diciembre de 2003. Comprobamos que todos ellos cumplían los criterios de Framingham para el diagnóstico de IC. Sólo tuvimos en cuenta los datos referentes al primer ingreso hospitalario, en el caso de que alguno de los pacientes reingresase durante el período de tiempo estudiado. En una base de datos recogimos los datos de filiación, clase funcional de la New York Heart Association (NYHA), etiología de la IC, datos ecocardiográficos, hemograma, etiología de la anemia, función renal, ionograma, mortalidad durante el ingreso, etc. Consideramos que un enfermo tenía anemia cuando el valor de la hemoglobina era inferior a $12 \mathrm{~g} / \mathrm{dL}$ e insuficiencia renal si la cifra de creatinina era mayor de $1,5 \mathrm{mg} / \mathrm{dL}$.

Para el análisis estadístico utilizamos el programa informático SPSS v 10.0. Comparamos las variables clínicas y demográficas de los enfermos mediante la prueba de la t de Student (variables cuantitativas) o de la Chi cuadrado y prueba de la exacta de Fisher (variables cualitativas). Utilizamos modelos de regresión logística para valorar los factores de pronósticos de mortalidad independientes. Se evaluó el efecto independiente de la anemia, la función renal y la clase funcional sobre la mortalidad

\section{RESULTADOS}

En total valoramos las historias clínicas de 272 enfermos cuyo diagnóstico principal era IC. Su edad media era 82,1 \pm 9 años y la mayoría eran mujeres (193 [71\%]). En el momento de su ingreso tenían anemia 106 pacientes $(39,9 \%)$ e insuficiencia renal 69 (25\%). Fallecieron 41 pacientes $(15 \%)$.
La mayor parte de los enfermos tenían una clase funcional II $(35,8 \%)$ o III $(52,8 \%)$ de la NYHA. La etiología más frecuente de IC fue la cardiopatía hipertensiva (34\%), seguido de cardiopatía isquémica (15\%), valvulopatía (14\%) y cor pulmonale (11\%). La etiología de la IC era desconocida en el $24,5 \%$ de los casos. En cuanto a la etiología de la anemia, en más de la mitad de los casos $(58,5 \%)$ no se estudio específicamente, el $16 \%$ se atribuyó a proceso crónico y el $15 \%$ a ferropenia. De los 69 enfermos con insuficiencia renal 40 (58\%) tenían anemia.

Cuando comparamos los enfermos que fallecieron $(n=41)$ con el resto de los pacientes con IC $(n=231)$ comprobamos que los primeros tenían unas cifras de hemoglobina y de hematocrito significativamente menores y unos niveles séricos de creatinina significativamente superiores (Tabla I). Obviamente, el porcentaje de enfermos con anemia o con insuficiencia renal fue también significativamente superior entre los pacientes que fallecieron. La asociación anemia e insuficiencia renal fue especialmente frecuente $(39 \%)$ entre los enfermos con IC que fallecieron. No hubo diferencias con respecto a la edad y el sexo (Tabla I).

En el análisis de regresión logística multifactorial la hemoglobina (odds ratio [OR] 0,78 por $\mathrm{g} / \mathrm{dL}$, intervalo de confianza [IC] 95\% 0,66- 0,923 p <0,01), la clase funcional de la NYHA (OR 2,2, IC 95\% 1,2-3,9 p < 0,01) y los niveles séricos de creatinina (OR 1,5 por $\mathrm{mg} / \mathrm{dL}$, IC $95 \% 0,98-2,31, \mathrm{p}=0,06$ ) constituyeron factores pronósticos independientes de mortalidad.

\section{TABLA I}

COMPARACIÓN DE DIVERSAS VARIABLES ENTRE LOS ENFERMOS CON IC QUE FALLECIERON CON RESPECTO A LOS QUE SOBREVIVIERON

\begin{tabular}{lllc}
\hline & $\begin{array}{l}\text { Enfermos con IC } \\
\text { que fallecieron } \\
(n=41)\end{array}$ & $\begin{array}{l}\text { Enfermos con IC } \\
\text { que sobrevivieron } \\
(n=231)\end{array}$ & $p$ \\
\hline Edad & $84,3 \pm 8$ & $81,7 \pm 9$ & 0,09 \\
Sexo (\% mujeres) & $65 \%(27 / 41)$ & $71 \%(166 / 231)$ & 0,43 \\
Estancia hospitalaria & $8,12 \pm 6$ & $7 \pm 4,3$ & 0,2 \\
Anemia (Hb $<12 \mathrm{~g} / \mathrm{dL})$ & & & \\
$\quad(\%)$ & $65 \%(27 / 41)$ & $36 \%(82 / 231)$ & $<0,001$ \\
Hemoglobina (g/dL) & $11,2 \pm 2,4$ & $12,6 \pm 2,1$ & $<0,001$ \\
Hematocrito $\%)$ & $34,3 \pm 7,6$ & $38,7 \pm 6,4$ & $<0,001$ \\
Insuficiencia renal & & & \\
$\quad(\mathrm{Cr}>1,5 \mathrm{mg} / \mathrm{dL})(\%)$ & $56 \%(23 / 41)$ & $19,4 \%(45 / 231)$ & $<0,001$ \\
$\begin{array}{c}\text { Creatinina (mg/dL) } \\
\text { Anemia }+ \text { insuficiencia }\end{array}$ & $1,7 \pm 0,78$ & $1,3 \pm 0,8$ & $<0,001$ \\
renal & $39 \%(16 / 41)$ & $10,3 \%(24 / 231)$ & $<0,001$ \\
\hline
\end{tabular}

\section{DISCUSIÓN}

Es llamativa la avanzada edad de los pacientes de nuestra serie (82,1 \pm 9 años) que refleja la realidad de los enfermos que ingresan en las plantas de Medicina Interna y que difiere claramente de los pacientes que se incluyen en los ensayos clínicos o que se valoran en las unidades de Cardiología (9). La precaria situación clínica de muchos de ellos, más de la mitad de los mismos con clase funcional III de la NYHA, justifica la elevada mortalidad observada. 
De nuestro estudio podemos deducir que un porcentaje considerable (casi el 40\%) de los enfermos que ingresan por IC tienen anemia. Este porcentaje es incluso superior al observado por otros autores $(2,6)$ y en parte puede estar relacionado con la mayor edad y peor situación clínica de nuestros pacientes. Cada vez es más evidente que la anemia es un factor pronóstico importante de mortalidad (2,3,7,9-11) y de reingreso hospitalario $(10,12)$. La nota discordante la aportan Kalra et al (13) en cuyo estudio la anemia no es un factor pronóstico independiente cuando en el análisis estadístico se incluye la edad y la creatinina sérica.

Son varias las causas que podrían justificar la anemia de la IC (hemodilución, malnutrición relacionada con la caquexia asociada a la IC avanzada, insuficiencia renal, inhibición de la proliferación hematopoyética por los inhibidores de la enzima conversora de la angiotensina, tratamiento antiagregante o anticoagulante, etc.) (1,7). Sin embargo, es posible que en muchos casos la etiología de anemia de la IC esté relacionada con la liberación de citocinas con capacidad de inhibir la eritropoyesis propia de las enfermedades crónicas $(2,14)$ y se relacione de forma estrecha con la edad de los enfermos y su situación funcional $(9,12)$. Resulta llamativo que en nuestra serie no se estudiase la causa de la anemia en más de la mitad de los casos, lo que refleja la escasa relevancia que los clínicos la otorgan en el contexto de un paciente anciano y con mala situación basal. No obstante, en los casos en los que se estudia las dos etiologías más frecuentes son la anemia asociada a proceso crónico y la ferropenia. Algo similar sucede en otras series (2).

La insuficiencia renal es otro factor pronóstico independiente de mortalidad en los enfermos con IC. Existe una clara relación entre IC, anemia e insuficiencia renal, ya que estos tres procesos son a la vez causa y consecuencia mutuas. Su asociación constituye el denominado síndrome cardiorrenalanemia $(15,16)$ que es especialmente frecuente entre los enfermos fallecidos en nuestro estudio. Este tipo de asociación se produce fundamentalmente en enfermos ancianos en los que el tratamiento médico debe ser personalizado y es necesaria una monitorización estrecha de la función renal y de los electrolitos (16) para evitar, al menos, reingresos hospitalarios. En la insuficiencia cardiorrenal existe un riesgo considerablemente aumentado de hiperpotasemia grave y de mayor deterioro de la función renal cuando se utilizan inhibidores de la enzima conversora de la angiotensina (IECAs) y/o espironolactona. Desde la publicación del estudio RALES (17) se ha generalizado el uso de la espironolactona en la IC, muchas veces asociado a los IECAs, y se ha observado como desde entonces ha aumentado de forma drástica la incidencia de hiperpotasemia grave en enfermos con IC, sobre todo en pacientes ancianos, con diabetes mellitus y/o con insuficiencia renal (18).

No sabemos si la asociación entre la anemia y el mal pronóstico de los enfermos con IC es causal o si la anemia es tan sólo un mero marcador de riesgo, como puede serlo la fracción de eyección. Sin embargo, parece evidente que la anemia debe agravar la situación clínica de los enfermos con IC, ya que se precisa aumentar el gasto cardíaco para aportar oxígeno a los tejidos, se favorece la isquemia miocárdica y se altera considerablemente la tolerancia y la capacidad al ejercicio $(1,19)$. A pesar de la estrecha relación entre la anemia, la insuficiencia renal avanzada y la IC, nuestros datos apoyan que la anemia per se es un factor independiente de mortalidad. Muy probablemente la corrección de la anemia puede mejorar la calidad de vida de estos enfermos, disminuir el número de hospitalizaciones y quizás mejorar su supervivencia (20-22), tal y como se ha demostrado en enfermos con insuficiencia renal (23). No obstante, son necesarios estudios de intervención prospectivos con un número suficiente de pacientes para confirmarlo.

En conclusión, la anemia es un hallazgo bastante frecuente de los enfermos con IC y constituye un factor de mal pronóstico independiente. La asociación de anemia con insuficiencia renal está especialmente relacionada con una mayor morbilidad y mortalidad y dificulta considerablemente el manejo clínico de estos pacientes.

\section{Bibliografía}

1. Parsi A, Kleber FX. Anaemia in heart failure: its diagnosis and management. Eur J Heart Fail 2003; 5: 3-4.

2. Ezekowitz JA, McAlister FA, Armstrong PW. Anemia is common in heart failure and is associated with poor outcomes: insights from a cohort of 12065 patients with new-onset heart failure. Circulation 2003; 107: 223-225.

3. Horwich TB, Fonarow GC, Hamilton MA, MacLellan WR, Borenstein $\mathrm{J}$. Anemia is associated with worse symptoms, greater impairment in functional capacity and a significant increase in mortality in patients with advanced heart failure. J Am Coll Cardiol 2002; 39: 1780-1786.

4. McClellan WM, Flanders WD, Langston RD, Jurkovitz C, Presley R. Anemia and renal insufficiency are independent risk factors for death among patients with congestive heart failure admitted to community hospitals: a population-based study. J Am Soc Nephrol 2002; 13: 19281936.

5. Silverberg DS, Wexler D, Iaina A. The importance of anemia and its correction in the management of severe congestive heart failure. Eur J Heart Fail 2002; 4: 681-686.

6. Urrutia A, Lupón J, González B, Parajón T, Altimir S, Coll R et al. Anemia y parámetros relacionados en pacientes de una unidad de insuficiencia cardiaca multidisciplinaria. Med Clin (Barc) 2004; 122: 121-125.

7. Androne AS, Katz SD, Lund L, LaManca J, Hudaihed A, Hryniewicz K et al. Hemodilution is common in patients with advanced heart failure. Circulation 2003; 107: 226-229.

8. Nissenson AR, Goodnough LT, Dubois RW. Anemia: not just an innocent bystander? Arch Intern Med 2003; 163: 1400-1404.

9. Szachniewicz J, Petruk-Kowalczyk J, Majda J, Kaczmarek A, Reczuch $\mathrm{K}$, Kalra PR et al. Anaemia is an independent predictor of poor outcome in patients with chronic heart failure. Int J Cardiol 2003; 90: 303-308.

10. Kosiborod M, Smith GL, Radford MJ, Foody JM, Krumholz HM. The prognostic importance of anemia in patients with heart failure. Am J Med 2003; 114: 112-119.

11. Mozaffarian D, Nye R, Levy WC. Anemia predicts mortality in severe heart failure: the prospective randomized amlodipine survival evaluation (PRAISE). J Am Coll Cardiol 2003; 41: 1933-1939.

12. Felker GM, Gattis WA, Leimberger JD, Adams KF, Cuffe MS, Gheorghiade $\mathrm{M}$ et al. Usefulness of anemia as a predictor of death and rehospitalization in patients with decompensated heart failure. Am J Cardiol 2003; 92: 625-628

13. Kalra PR, Collier T, Cowie MR, Fox KF, Wood DA, Poole-Wilson PA et al. Haemoglobin concentration and prognosis in new cases of heart failure. Lancet 2003; 362: 211-212.

14. Sharma R, Coats AJS, Anker SD. The role of inflammatory mediators in chronic heart failure: cytokines, nitric oxide, and endothelin-1. Int J 
Cardiol 2000; 72: 175-186

15. Silverberg D, Wexler D, Blum M, Wollman Y, Iaina A. The cardio-renal anaemia syndrome: does it exist? Nephrol Dial Transplant 2003; 18 (Suppl 8): 7-12.

16. Caramelo C, Gil P. Insuficiencia combinada cadiorrenal: una entidad emergente. Med Clin (Barc) 2003; 121: 710-717.

17. Pitt B, Zannad F, Remme WJ, Cody R, Castaigne A, Perez A et al. The effect of spironolactone on morbidity and mortality in patients with sever heart failure. N Engl J Med 1999; 341: 709-717.

18. Juurlink DN, Mamdani MM, Lee DS, Kopp A, Austin PC, Laupacis A et al. Rates of hyperkalemia after publication of the Randomized Aldactone Evaluation Study. N Engl J Med 2004; 351: 543-551.

19. Kalra PR, Bolger AP, Francis DP, Genth-Zotz S, Sharma R, Ponikowski $\mathrm{PP}$ et al. Effect of anemia on exercise tolerance in chronic heart failure in men. Am J Cardiol 2003; 91: 888-891.

20. Silverberg DS, Wexler D, Blum M, Keren G, Sheps D, Leibovitch E et al. The use of subcutaneous erythropoietin and intravenous iron for the treatment of the anemia of severe, resistant congestive heart failure improves cardiac and renal function and functional cardiac class, and markedly reduces hospitalizations. J Am Coll Cardiol 2000; 35: 1737 1744.

21. Silverberg DS, Wexler D, Sheps D, Blum M, Keren G, Baruch R et al. The effect of correction of mild anemia in severe, resistant congestive heart failure using subcutaneous erythropoietin and intravenous iron: a randomized controlled study. J Am Coll Cardiol 2001; 37: 17751780 .

22. Silverberg DS, Wexler D, Blum M, Tchebiner JZ, Sheps D, Keren G et al. The effect of correction of anaemia in diabetics and non-diabetics with severe resistant congestive heart failure and chronic renal failure by subcutaneous erythropoietin and intravenous iron. Nephrol Dial Transplant 2003; 18: 141-146.

23. Jones M, Ibels L, Schenkel B, Zagari M. Impact of epoetin alfa on clinical end points in patients with chronic renal failure: analysis. Kidney Int 2004; 65: 757-767. 